\title{
Diacronie
}

Studi di Storia Contemporanea

$\mathrm{N}^{\circ} 20,4 \mid 2014$

II diritto militante

\section{Prefazione n. 20 - dicembre 2014}

Jacopo Bassi e Deborah Paci

\section{(2) OpenEdition}

\section{Journals}

\section{Edizione digitale}

URL: http://journals.openedition.org/diacronie/1662

DOI: 10.4000/diacronie.1662

ISSN: 2038-0925

\section{Editore}

Association culturelle Diacronie

\section{Notizia bibliografica digitale}

Jacopo Bassi e Deborah Paci, « Prefazione n. 20 - dicembre 2014 », Diacronie [Online], № 20, 4| 2014, Messo online il 01 décembre 2014, consultato il 23 septembre 2020. URL : http://

journals.openedition.org/diacronie/1662; DOI : https://doi.org/10.4000/diacronie.1662

Questo documento è stato generato automaticamente il 23 settembre 2020.

Creative Commons License 
Prefazione n. 20 - dicembre 2014

Jacopo Bassi e Deborah Paci

IL NUMERO 20... CINQUE ANNI DOPO 
1 Cinque anni fa, nel novembre del 2009, nasceva la rivista «Diacronie. Studi di storia contemporanea». Solo alcuni mesi prima un gruppo di studenti, accomunati dall'esperienza di studio nel dipartimento di Storia dell'Università di Bologna, decideva di realizzare uno spazio online per dare visibilità ai lavori di ricerca portati avanti individualmente; il

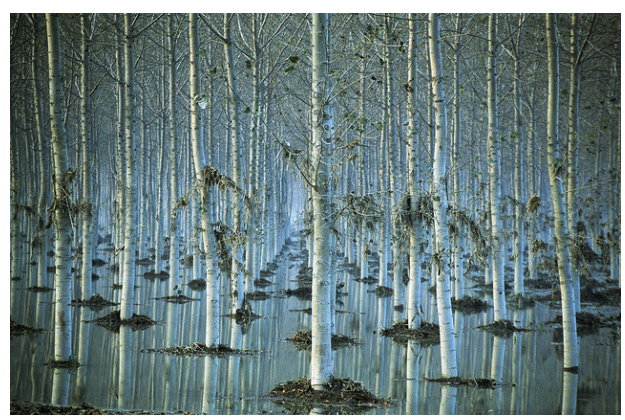
progetto, successivamente, si sviluppava e approdava all'idea di dar vita ad un luogo di confronto e di dibattito. Nasceva così l'idea della rivista: il primo numero aveva come filo conduttore il tema dei confini; su questo argomento, a distanza di 20 anni dalla caduta del muro di Berlino, i redattori della rivista decisero di confrontarsi.

2 A partire da questa prima esperienza è maturata la consapevolezza - cresciuta insieme al numero dei fascicoli della rivista - di poter dar vita ad un'occasione di confronto e di diffusione delle conoscenze in ambito storico. L'essere sorti in maniera indipendente e autonoma dal mondo accademico - pur collaborando e dibattendo con questo - ci ha consentito di mantenere un'assoluta libertà decisionale; una libertà che coincide con la piena assunzione di responsabilità per la linea editoriale e per gli errori nei quali inevitabilmente - si incappa. La stessa libertà che pesa ogni volta in cui ci si confronta con il lavoro redazionale, portato avanti volontariamente nel corso di questi anni dai soli redattori e senza sostegni, né economici, né istituzionali. Per questo diciamo grazie a chi, in questi anni, ha fatto parte della redazione e ha fornito il proprio tempo, le proprie competenze, le proprie energie e la propria passione per la realizzazione di questo progetto; esprimiamo la massima riconoscenza nei confronti di tutti i nostri collaboratori, sempre disponibili a fornirci il loro prezioso aiuto; ringraziamo il comitato scientifico, i docenti, i ricercatori e gli autori che hanno partecipato a vario titolo ad ognuno dei singoli numeri.

Dopo cinque anni dalla registrazione della rivista, siamo stati riconosciuti dall'ANVUR come pubblicazione scientifica, siamo stati inseriti tra le riviste presenti sulla piattaforme DOAJ e su Dialnet, siamo approdati - e presto saremo online - su revues.org e facciamo parte delle riviste catalogate sull'ACNP.

4 Utilizzare un supporto immateriale per la pubblicazione ci ha consentito di accorciare distanze e tempi, rendendo possibile la diffusione di conoscenze provenienti da altre parti del mondo e da altre esperienze di studio; ci ha permesso di non doverci porre il problema degli spazi e dei costi, gravame che avrebbe certamente ucciso il progetto nella culla. Scegliere di pubblicare su internet è stata una piacevole necessità, che ci ha portato ad aprirci ad alcune esperienze internazionali di collaborazione (quella con le riviste brasiliane «Chronidas» e «Cadernos do Tempo Presente» e quella francese «Etudes Corses») e di blogging (Devenir historien-ne). Tutte queste esperienze nascono da contatti coltivati nel tempo, sviluppati e divenuti patrimonio comune della rivista e dei lettori. La stessa redazione di Diacronie è l'espressione della diaspora comune a tutti coloro che decidono di proseguire l'esperienza di ricerca nel mondo attuale. Questo allontanarsi si è così trasformato nell'opportunità di stringere relazioni e ampliare l'orizzonte delle proprie conoscenze: i singoli membri della redazione, pur essendo distanti fisicamente, fanno rete per diffondere la ricerca. Se la stanzialità e la stabilità 
lavorativa sono dunque utopie irrealizzabili nell'epoca attuale, abbiamo deciso di puntare sulla continuità delle relazioni, sulla loro moltiplicazione e sulla libera diffusione delle conoscenze offerte dalla rete.

Abbiamo cercato di far corrispondere a quanto realizzato in forma virtuale anche un'applicazione pratica: Diacronie ha realizzato diversi incontri sul tema della Digital History (in collaborazione con l'European Association of Digital Humanities, l'Università Italo Francese, il Cursus intégré franco-italien d'Histoire et civilisations comparées, il Collegio Superiore, l'Université Paris 7 e l'Università di Bologna). Una delle nostre speranze, quando mettemmo in piedi questo progetto, era quella di ridurre la distanza tra i luoghi di formazione dello storico (i corsi di laurea e i master) e gli spazi dedicati alla diffusione della ricerca. Non siamo in grado di poter dire di esserci riusciti, ma certamente di averci provato e di volerci provare.

Proprio a partire da alcune delle premesse da cui è nata «Diacronie. Studi di storia contemporanea», riproponiamo un secondo Laboratorio, concepito come spazio di pubblicazione per i lavori svolti dagli studenti dei corsi di Laurea Magistrale; la speranza è sempre quella che esperienze come Diacronie possano moltiplicarsi e che si diffonda un numero sempre maggiore di spazi di confronto e di discussione e auspicabilmente - riviste indipendenti, che consentano di ridurre la distanza fra chi scrive e chi legge, senza per questo perderne in scientificità o in serietà.

Buona lettura.

\section{AUTORI}

\section{JACOPO BASSI}

Nel 2006 consegue la Laurea Triennale in « Storia del mondo contemporaneo » presso l'Università di Bologna sostenendo una tesi in Storia e istituzioni della Chiesa ortodossa dal titolo Tra Costantinopoli e Atene: Il passaggio delle diocesi dell'Epiro all'amministrazione della Chiesa di Grecia e la 'Praxis' del 1928, relatore il Professor Enrico Morini. Nel 2007, nel quadro del programma di scambio Erasmus, ha frequentato per un trimestre l'École Normale Supérieure (ENS) di Parigi; ha effettuato un periodo di soggiorno durante i mesi di gennaio e febbraio 2008 presso l' École Française d' Athènes, sotto il tutorato del dottor Anastassios Anastassiadis, membro del comitato scientifico di questa istituzione. Nel luglio 2008 ha discusso la Tesi Specialistica in Storia della Chiesa - relatore il Professor Umberto Mazzone, correlatore il Professor Enrico Morini - dal titolo Epiro crocifisso o liberato? La Chiesa ortodossa in Epiro e in Albania meridionale nel XX secolo (1912-1967). Ha lavorato come redattore per la casa editrice L'Inventaire e ha curato il progetto Dictionnaire universel des femmes créatrices - secteur "Femmes du livre" in corso di pubblicazione presso Editions des Femmes. Attualmente collabora con la casa editrice Il Mulino alla creazione dell'archivio digitale dei libri del Mulino, Darwinbooks e con la casa editrice Carocci. 


\section{DEBORAH PACI}

Nel 2006 consegue la Laurea Triennale in «Storia contemporanea» presso l'Università di Bologna discutendo una tesi sulla battaglia autonomista condotta dal Partito d'Azione in Valle d'Aosta e in Sicilia durante il triennio 1943-1946, dal titolo Il contributo del Partito d'Azione nella lotta per le autonomie. Sicilia e Valle d'Aosta a confronto (1943-1946). Nel 2007 partecipa al programma internazionale «Cursus intégré franco-italien d' histoire européenne comparée», promosso dall'Università di Bologna e dall'Université Paris VII - Denis Diderot. Nel 2008 consegue un doppio titolo di Laurea Specialistica in «Storia d'Europa» e di Master 2 «Histoire et civilisations comparées», sostenendo una tesi sulla ricezione del pensiero di Pierre-Joseph Proudhon presso i fuorusciti italiani in Francia dal titolo Dall'anarchia al federalismo. La tradizione proudhoniana nei Fuorusciti italiani in Francia. L'attenzione in questo studio è stata rivolta alla lettura delle teorie proudhoniane operata dal sociologo Georges Gurvitch e alla sua trasmissione negli ambienti del fuoruscitismo italiano negli anni tra le due guerre. Tra il 2009 e il 2011 è stata borsista presso l'Ecole Française de Rome e visiting student presso l'University of Malta. Nel 2013 consegue un dottorato di ricerca in cotutela in «Scienze storiche» presso l'Università di Padova e in «Histoire» presso Université de Nice Sophia-Antipolis, svolgendo una ricerca sul mito del Risorgimento mediterraneo e sul progetto imperialista fascista in Corsica e a Malta negli anni tra le due guerre. Fa parte del Centro Interuniversitario di Storia Culturale (CSC) e del Centre de la Méditerranée Moderne et Contemporaine (CMMC). Attualmente Assegnista di ricerca presso l'Università Ca' Foscari di Venezia sta conducendo uno studio sulle politiche identitarie e sull'immaginario insulare nelle isole del Mediterraneo e del Baltico. 